Swarthmore College

Works

$5-1-2000$

\title{
Helix Inversion In The Chiral Nematic And Isotropic Phases Of A Liquid Crystal
}

\author{
Benjamin P. Huff , '99 \\ Jacob J. Krich , '00 \\ Peter J. Collings \\ Swarthmore College, pcollin1@swarthmore.edu
}

Follow this and additional works at: https://works.swarthmore.edu/fac-physics

Part of the Physics Commons

Let us know how access to these works benefits you

\section{Recommended Citation}

Benjamin P. Huff , '99; Jacob J. Krich , '00; and Peter J. Collings. (2000). "Helix Inversion In The Chiral Nematic And Isotropic Phases Of A Liquid Crystal". Physical Review E. Volume 61, Issue 5. 5372-5378. DOI: 10.1103/PhysRevE.61.5372

https://works.swarthmore.edu/fac-physics/268

This work is brought to you for free by Swarthmore College Libraries' Works. It has been accepted for inclusion in Physics \& Astronomy Faculty Works by an authorized administrator of Works. For more information, please contact myworks@swarthmore.edu. 


\title{
Helix inversion in the chiral nematic and isotropic phases of a liquid crystal
}

\author{
Benjamin P. Huff, Jacob J. Krich, and Peter J. Collings \\ Department of Physics and Astronomy, Swarthmore College, Swarthmore, Pennsylvania 19081 \\ (Received 27 September 1999; revised manuscript received 10 December 1999)
}

\begin{abstract}
Measurements of the chirality $(2 \pi /$ pitch $)$ in the chiral nematic phase and of a structural constant proportional to the chirality in the isotropic liquid for a system in which a helix inversion line crosses the chiral nematic to isotropic phase transition line are reported. While the chirality shows a strong temperature dependence in the chiral nematic phase, it loses all temperature dependence in the isotropic phase. In addition, the chirality in the isotropic phase is proportional to the chirality in the chiral nematic phase at the phase transition, and may in fact be continuous across the transition. While molecular field and phenomenological theories can explain the strong temperature dependence in the chiral nematic phase, including the helix inversion, these theories predict a strong discontinuity in the chirality at the phase transition that is not supported by experiment. So while a theory that includes short range molecular correlations is called for to understand the behavior of the chirality across the phase transition, theoretical attempts to explain the chirality of a phase from a microscopic level must account for the strong role played by long range orientational order.
\end{abstract}

PACS number(s): 61.30.-v, 64.70.Md

\section{INTRODUCTION}

The origin of chirality and the transfer of chirality from the molecules to an anisotropic phase have been the subjects of recent interest. While it has long been known that chiral molecules tend to form chiral liquid cystal phases and that small amounts of a chiral substance added to an achiral liquid crystal produce chiral phases, the recent interest stems from a desire to understand these effects at the microscopic level. Theoretical calculations, both classical and quantum mechanical, have been performed over the last 25 years [1-8], but the inability of the older theories to describe the complicated behavior uncovered in more recent experiments has been the driving force behind the latest theoretical work.

One recent experimental finding that demonstrates the wealth of behavior that must be explained starting at the microscopic level is helix inversion in chiral nematic liquid crystals [9-17]. The chiral nematic phase is characterized by a preferred direction for molecular orientation (called the director) that rotates in helical fashion throughout the phase. The distance over which the director rotates through $2 \pi$ radians is called the pitch, which can be as small as $0.1 \mu \mathrm{m}$ or as large as allowed by the container. The chirality of the phase $q_{0}$ is defined to be $2 \pi$ divided by the pitch. Helix inversion occurs when the chirality of a helix of one handedness decreases with increasing temperature, becomes zero at a certain temperature, and then increases with increasing temperature with a helix of the opposite handedness. Two general explanations for this behavior have been suggested. In one, the statistical distribution of the many molecular conformations shifts with changing temperature $[10,13]$. In the other, the competition between the different temperature dependences and opposite chiralities of multiple chiral centers causes the helix inversion $[9,12]$. Experimental support for the first explanation comes from nematic systems with chiral dopants $[11,16]$, whereas experimental results verifying the second explanation use diastereomeric liquid crystalline compounds $[14,15]$.

Due to the sensitivity of the chirality to temperature changes, helix inversion compounds are excellent systems in which to probe the microscropic origin of the chirality in liquid crystalline phases. While the experimental evidence indicates that a very complex interplay between molecular structure and intermolecular interactions is responsible for the chirality of the chiral nematic phase, the theoretical results corroborate this by demonstrating that the interactions are dependent on both long-range orientational order and short-range molecular correlations. Since dramatic changes in the long-range orientational order occur at some phase transitions, one way to investigate the influence of longrange order on the origin of chirality is to investigate the effect of crossing a phase transition when a helix inversion is nearby. If the short-range molecular correlations are dominant in determining the chirality of the phase, then the temperature dependence of the chirality should show little change as the phase transition is crossed. If the long-range orientational order dominates the interactions responsible for the chirality, then one should see a dramatic difference in the chiral behavior on either side of the phase transition.

A quick review of two mean field theories is first presented, to show (1) that they can explain helix inversion in the chiral nematic phase and (2) that they predict a temperature-independent chirality in the isotropic phase. Second, experimental evidence on a system in which the helix inversion temperature can be moved to the transition between the chiral nematic and isotropic liquid phases is described. Third, chirality measurements in the chiral nematic phase and optical activity measurements in the isotropic phase are used to investigate the chirality on both sides of the phase transition. These experiments show that the chirality is strongly temperature dependent in the chiral nematic phase and temperature independent in the isotropic phase. In contradiction to the predictions of the two mean field theories, the constant chirality in the isotropic phase is proportional to the chirality in the chiral nematic phase at the phase transition and may in fact be continuous across the transition. Thus these experimental results demonstrate the importance of the long-range orientational order in the origin of chirality in 
liquid crystal phases, while strongly suggesting that a theory including short-range molecular correlations is necessary for even a qualitative understanding of chiral liquid crystalline systems.

\section{THEORY}

There are two general theoretical approaches to understanding the nematic phase that have proven to be highly informative for many years, the molecular statistical approach represented by the Maier-Saupe theory [18] and the phenomenological approach represented by Landau-de Gennes theory [19]. These two theories have been the starting point for a myriad of modifications and extensions during the latter part of this century, with many of these aiding the understanding of liquid crystal phases and phase transitions. The molecular statistical approach starts with a molecular field potential that depends on at least one order parameter. Since the order parameter itself is a statistical quantity, a self-consistent equation can be generated with the order parameter being the only unknown. Solving this equation gives the order parameter as a function of other variables, for example, temperature. The phenomenological approach begins with the realization that near the phase transition to the isotropic liquid, the order parameter of the nematic phase is small. This allows the free energy to be expanded in a power series of the order parameter, with only those terms allowed by symmetry appearing. Finding the minimum of the free energy for a set of variables yields the value of the order parameter.

Since both of these theories pertain to achiral systems, they must be modified to include chirality. In the extension of the Maier-Saupe theory proposed by van der Meer et al. [1], cylindrically symmetric chiral molecules interact via a potential based on the electric multipole expansion, producing a molecular field potential containing two order parameters $\left\langle P_{2}(\cos \theta)\right\rangle$ and $\left\langle P_{4}(\cos \theta)\right\rangle$, where $P_{2}$ and $P_{4}$ are the second and fourth Legendre polynomials, respectively, $\theta$ is the angle the long axis of the molecule makes with the director, and the brackets denote a thermal average. Minimizing the resulting free energy with respect to the two order parameters and the chirality $q_{0}$ yields values for all three quantities. The expression for the chirality is

$$
q_{0}=q_{I}\left[\frac{1+a\left(\frac{\left\langle P_{4}\right\rangle}{\left\langle P_{2}\right\rangle}\right)^{2}}{1+b\left(\frac{\left\langle P_{4}\right\rangle}{\left\langle P_{2}\right\rangle}\right)^{2}}\right],
$$

where $q_{I}, a$, and $b$ are constants that depend on the strength of the various intermolecular interactions and the average distance between molecules. Since the ratio of the two order parameters appearing in Eq. (1) goes to zero as each approaches zero, the parameter $q_{I}$ is the chirality in the isotropic phase and is temperature independent. In principle, $\left\langle P_{2}\right\rangle$, $\left\langle P_{4}\right\rangle$, and $q_{0}$ should be determined self-consistently. However, since $q_{0}$ times the average distance between molecules is very small, the $q_{0}$ dependence of $\left\langle P_{2}\right\rangle$ and $\left\langle P_{4}\right\rangle$ should be extremely weak. To a good approximation, therefore, $\left\langle P_{2}\right\rangle$ and $\left\langle P_{4}\right\rangle$ can be found via the conventional Maier-Saupe theory and these values substituted in Eq. (1) to find $q_{0}$. Of course, the values for $q_{I}, a$, and $b$ are unknown, so all that can be done is to determine them by fitting the theory to experimental data.

The phenomenological theory can be extended to include chirality by simply adding terms allowed by symmetry to the conventional free energy expression. There are several terms that are good candidates, but knowing which ones are likely to dominate is difficult to ascertain. Since the results are qualitatively the same regardless of which terms are added, one example is given here. It is based on assuming that the chirality in a simple extension of the phenomenological theory depends linearly on the order parameter. The free energy in a chiral system can be written as [20]

$$
f=\frac{1}{3}\left[a_{0}\left(T-T^{*}\right)-\frac{3}{4} b q_{0}^{2}\right] S^{2}-\frac{2}{27} \mu S^{3}+\frac{1}{9} \lambda S^{4},
$$

where $a_{0}, b, \mu$, and $\lambda$ are temperature independent constants, $T^{*}$ is a second order transition temperature, $S$ is the order parameter, and $q_{0}$ is the chirality. If the chirality $q_{0}$ is allowed to depend linearly on $S$ as follows:

$$
q_{0}=q_{I}(1+\alpha S),
$$

then the free energy can be minimized as a function of temperature for any set of parameters $T^{*}, a_{0}, b, \mu, \lambda, q_{I}$, and $\alpha$. Notice that this form of $q_{0}$ introduces terms in the free energy of the form $q_{I}^{2} S^{2}, q_{I}^{2} S^{3}$, and $q_{I}^{2} S^{4}$. Notice also that $q_{I}$ is the temperature independent chirality of the isotropic phase since $S$ is zero in the isotropic phase. Since the parameters in the free energy are not known, they must be determined by a fit to experimental data.

\section{EXPERIMENT}

Three liquid crystals were used in this study. The helix inversion compound, 4-[(S,S)-2,3-epoxyhexyloxy]-phenyl-4(decyloxy)-benzoate or $(S, S)$-EPHDBPE, was purchased from Aldrich and used without further purification. The liquid crystal added to (S,S)-EPHDBPE to move the helix inversion to the transition to the isotropic phase was cholesteryl myristate $(\mathrm{CM})$. It was purchased from Kodak and recrystallized several times in ethanol. Finally, a chiral compound, S-4-(2-methylbutyl)phenyl-4-decyloxybenzoate (CE6), available both as a pure entaniomer and as a racemic mixture, was used to verify a theoretical prediction crucial to this investigation. Both forms of CE6 were purchased from EM Chemicals and used without further purification. All three of these compounds along with their transition temperatures are shown in Fig. 1.

The chiral nematic phase of $(S, S)$-EPHDBPE exists from 78 to $96^{\circ} \mathrm{C}$ with a helix inversion at $83^{\circ} \mathrm{C}$. At temperatures below the helix inversion, the chiral nematic structure is lefthanded, while above the helix inversion the structure is right handed. Since CM forms a left-handed chiral nematic structure and possesses a transition to the isotropic phase at a lower temperature than $(S, S)$-EPHDBPE, adding small amounts of CM to $(S, S)$-EPHDBPE causes the helix inversion temperature to increase and the transition to the isotropic phase to decrease. As shown in Fig. 2, the helix inversion 
4-[(S,S)-2,3-epoxyhexyloxy]-phenyl-4-(decyloxy)-benzoate (S,S)-EPHDBPE
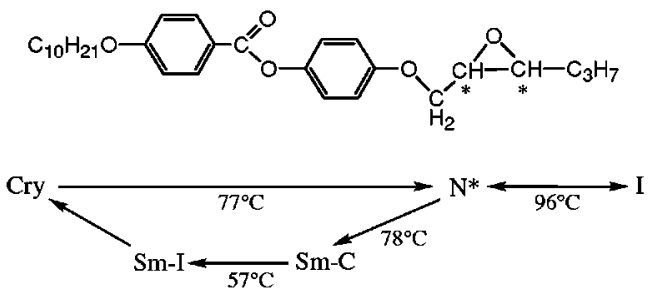

Cholesteryl Myristate (CM)
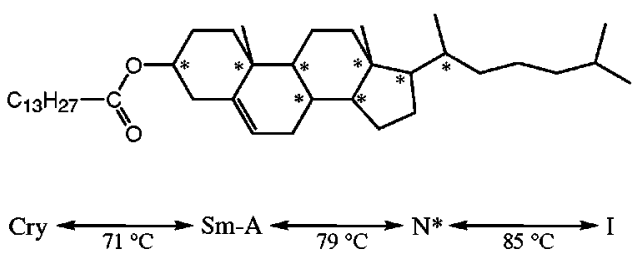

S-4-(2-methylbutyl)phenyl 4-decyloxybenzoate (CE6)
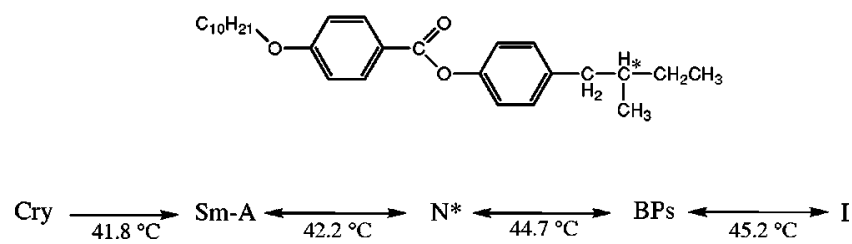

FIG. 1. Structure and phase transition temperatures for the materials used in this investigation. Cry: crystal, Sm-I: smectic-I, Sm-C: smectic-C, $\mathrm{N}^{*}$ : chiral nematic, BPs: blue phases, I: isotropic.

line and isotropic transition line meet at about $12 \mathrm{wt} . \% \mathrm{CM}$ in $(S, S)$-EPHDBPE.

To measure the chirality in the chiral nematic phase, the sample was introduced into a $10 \mu \mathrm{m}$ glass cell with rubbed polyimide surfaces. The resulting planar texture with an integer number of half twists of the helix appeared uniform

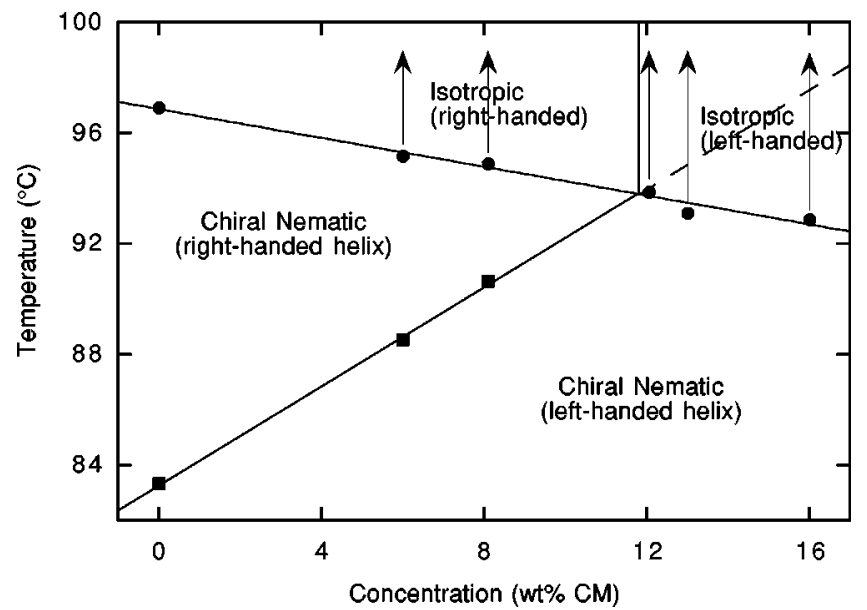

FIG. 2. Phase diagram of the CM-EPHDBPE system. filled circles: chiral nematic-isotropic transition. filled squares: helix inversion. The lines are linear least square fits to the data. The arrows denote where optical activity measurements were made. The helix inversion line is vertical in the isotropic phase. and highly colored under a polarizing microscope, except for a temperature interval surrounding the helix inversion where the sample appeared black. As the temperature was varied and the chirality changed, the number of half twists of the helix would change by one as disclinations passed across the field of view. Since the free energy densities of two textures are equal as the disclination separating the two textures passes, this allows the "natural" chirality of the sample to be measured. If there are $n$ half twists across a space $d$, then the "constrained" chirality $q$ is given by

$$
q=\frac{2 \pi}{\text { pitch }}=\frac{2 \pi}{2 d / n}=n \frac{\pi}{d} .
$$

The free energy density of a texture with "constrained" chirality $q$ when only twist distortion is present can be expressed as

$$
f=-k_{2} q+\frac{1}{2} k_{22} q^{2}
$$

where $k_{2}$ and $k_{22}$ are twist elastic constants [21]. The "natural' chirality $q_{0}$ is found by minimizing $f$,

$$
q_{0}=\frac{k_{2}}{k_{22}} \text {. }
$$

Subsituting $q_{0} k_{22}$ for $k_{2}$ and either $n \pi / d$ or $(n+1) \pi / d$ for $q$, and then equating $f / k_{22}$ for textures with $n$ and $(n+1)$ half twists, leads to a relation that can be used to determine the "natural" chirality $q_{0}$ when the disclination passes

$$
q_{0}=\frac{\pi}{d}(2 n+1)
$$

By keeping track of how many disclinations have passed since the helix inversion, the number of half twists $n$ is known. Of course the heating and cooling must be done extremely slowly, so the sample remains close to equilibrium as the disclination moves across the field of view. In addition, to measure accurately the thickness of the cell $d$, interferometric methods were used [22]. This method of measuring chirality was tested by comparison with chirality measurements utilizing the Cano lines in a wedge shaped cell, and the results from both methods were always consistent.

Optical activity measurements in the isotropic phase were obtained using a $1 \mathrm{~cm}$ path length glass cell. The system is capable of measuring optical rotation angles with an accuracy of one millidegree and has been described elsewhere [23]. In all cases the sample was heated just above the temperature of the chiral nematic to isotropic transition and then optical rotation angles were measured over a $20-30^{\circ} \mathrm{C}$ interval in the isotropic phase in steps of $0.2-0.25^{\circ} \mathrm{C}$. Temperature homogeneity across the sample was better than $0.1^{\circ} \mathrm{C}$.

\section{RESULTS}

Chirality measurements on various mixtures of $\mathrm{CM}$ in $(S, S)$-EPHDBPE allowed the phase diagram shown in Fig. 2 to be determined and produced data on chirality as a function 


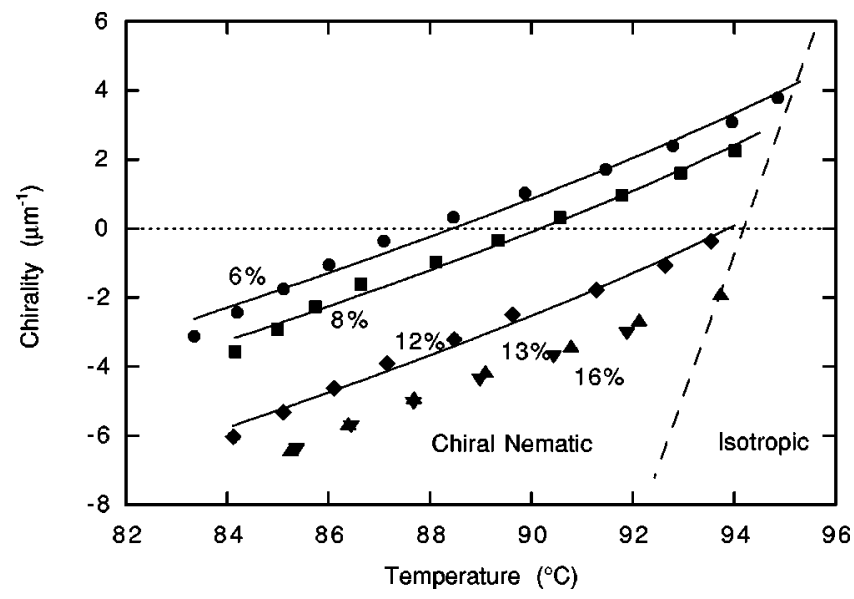

FIG. 3. Chirality measurements in the chiral nematic phase for five CM-EPHDBPE mixtures. The lines are least square fits to the extended Maier-Saupe theory with $b=0$.

of temperature in mixtures of $0.0,6.0,8.1,12.1,13.0$, and 16.0 wt. \% CM in $(S, S)$-EPHDBPE. These data are shown in Figs. 3 and 4 along with least square fits to the extended Maier-Saupe and Landau-de Gennes theories, respectively. Notice that the data, which includes an actual inversion in some cases, agrees with the theory if the proper set of parameters is chosen.

In the fits to the extended Maier-Saupe theory, the parameter $b$ has been set to zero to keep things as simple as possible. It should be pointed out, however, that from the standpoint of the electric multipole expansion, there is no reason to suspect that $b$ should be much smaller than $a$.

In addition, reasonable values for the phenomenological parameters have been assigned for the fits to the Landau-de Gennes theory using the following strategy. If $q_{0}=0$ in Eq. (2), then the temperature dependence of the order parameter for a nematic liquid crystal can be written as

$$
S=\frac{3}{4} S_{C}\left[1+\frac{1}{3} \sqrt{1-8 \frac{T-T_{C}}{T_{C}-T^{*}}}\right],
$$

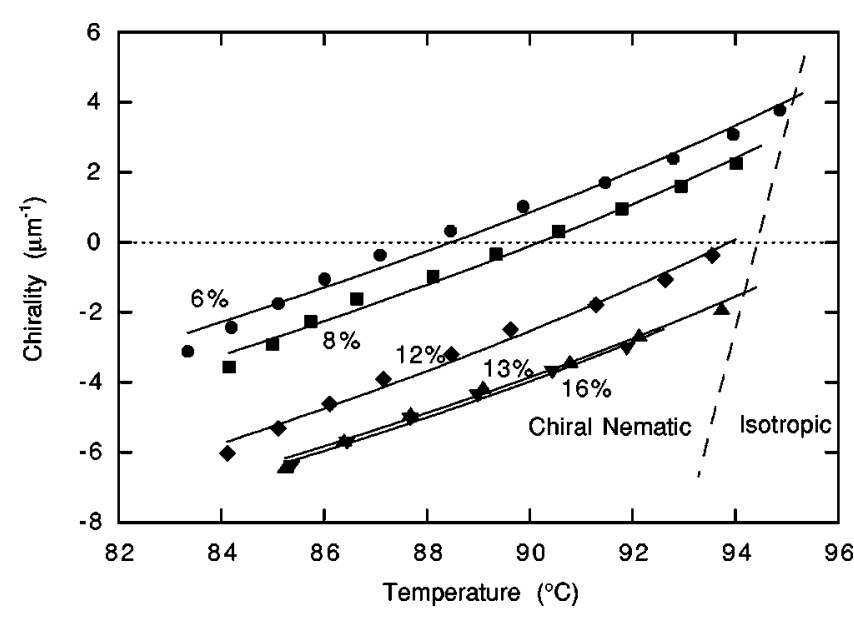

FIG. 4. Chirality measurements in the chiral nematic phase for five CM-EPHDBPE mixtures. The lines are least square fits to the extended Landau-de Gennes theory with $b / a_{0}=1.8 \times 10^{-11} \mathrm{~cm}^{2} \mathrm{~K}$, $\mu / a_{0}=180 \mathrm{~K}$, and $\lambda / a_{0}=160 \mathrm{~K}$.
TABLE I. Least square fit parameters using the extended MaierSaupe theory for four of the six CM- $(S, S)$-EPHDBPE mixtures investigated. For simplicity, the parameter $b$ was set to zero for all fits.

\begin{tabular}{ccc}
\hline \hline wt. \% CM-EPHDBPE & $q_{I}\left(\mu \mathrm{m}^{-1}\right)$ & \multicolumn{1}{c}{$a$} \\
\hline 0.0 & $36.4 \pm 7.9$ & $-8.04 \pm 0.06$ \\
6.0 & $17.6 \pm 2.4$ & $-9.72 \pm 0.17$ \\
8.1 & $15.8 \pm 2.8$ & $-10.57 \pm 0.22$ \\
12.1 & $13.3 \pm 3.0$ & $-12.73 \pm 0.64$ \\
13.0 & & \\
16.0 & & \\
\hline \hline
\end{tabular}

where $S_{C}$ is the transition value of the order parameter

$$
S_{C}=\frac{1}{3} \frac{\mu}{\lambda}
$$

and where $T_{C}$ is the phase transition temperature. Values for $S_{C}$ and $T_{C}-T^{*}$ that give good agreement with the order parameter measurements in 4- $n$-pentyl- $4{ }^{\prime}$-cyanobiphenyl (5CB) [24], a typical nematic liquid crystal, are 0.38 and 7.8 $\mathrm{K}$, respectively. Values of $\mu / a_{0}$ and $\lambda / a_{0}$ are then determined by

$$
\frac{\mu}{a_{0}}=\frac{9\left(T_{C}-T^{*}\right)}{S_{C}}
$$

and

$$
\frac{\lambda}{a_{0}}=\frac{3\left(T_{C}-T^{*}\right)}{S_{C}^{2}} .
$$

The parameter $b / a_{0}$ can be determined by realizing it is related to the correlation length in the isotropic phase at the phase transition $\xi_{C}$, which measurements show is about equal to $15 \mathrm{~nm}$ [25]

$$
\frac{b}{a_{0}}=\xi_{C}^{2}\left(T_{C}-T^{*}\right) .
$$

It should be noted that the temperature dependence of the order parameter in the fits to the data depends on the values of $b / a_{0}, q_{I}$, and $\alpha$, but the effect is quite weak (a decrease of about $4 \%$ compared to the data from $5 \mathrm{CB}$ ).

Whether $b$ is set to zero or not or whether or not the phenomenological parameters are varied from the assigned values, good agreement between the theories and the data in the chiral nematic phase is possible. More important, as Tables I and II show, in all cases the chirality jumps to a signigicantly higher value in the isotropic phase according to both theories. The fact that these theories cannot predict a continuous chirality across the transition or even a proportionality in the chirality on either side of the transition, regardless of the parameters used, is evident from Eqs. (1) and (3), where the discontinuity in the order parameters forces a non-proportional discontinuity in the chirality.

The optical activity measurements in the isotropic phase for five mixtures of CM in $(S, S)$-EPHDBPE are shown in Fig. 5. The region of the phase diagram in which these mea- 
TABLE II. Least square fit parameters using the extended Landau-de Gennes theory for the six CM- $(S, S)$-EPHDBPE mixtures investigated. Fixed values for the following parameters were used in all the fits: $b / a_{0}=1.8 \times 10^{-11} \mathrm{~cm}^{2} \mathrm{~K}, \mu / a_{0}=180 \mathrm{~K}$, and $\lambda / a_{0}=160 \mathrm{~K}$.

\begin{tabular}{ccc}
\hline \hline wt. \% CM-EPHDBPE & $q_{I}\left(\mu \mathrm{m}^{-1}\right)$ & $\alpha$ \\
\hline 0.0 & $25.8 \pm 0.1$ & $-1.64 \pm 0.01$ \\
6.0 & $13.2 \pm 0.7$ & $-1.85 \pm 0.02$ \\
8.1 & $11.7 \pm 0.8$ & $-2.03 \pm 0.02$ \\
12.1 & $9.42 \pm 0.95$ & $-2.53 \pm 0.08$ \\
13.0 & $7.6 \pm 1.3$ & $-2.91 \pm 0.19$ \\
16.0 & $5.7 \pm 1.7$ & $-3.53 \pm 0.49$ \\
\hline \hline
\end{tabular}

surements were taken are indicated in Fig. 2. Two findings are immediately clear from the data. First, there are two contributions to the optical activity in this system. There is a "structural" part that stems from the short range order in the isotropic phase. This contribution decreases with increasing temperature and becomes insignificant about $25^{\circ} \mathrm{C}$ above the transition. There is also a "molecular'" part that is fairly constant with temperature but does show a small temperature dependence. Second, the "structural" component decreases the closer the CM concentration is to about 12 wt. \%, the point at which the helix inversion line meets the isotropic transition line, and in fact is almost zero in the $12.1 \mathrm{wt} \%$ mixture. Furthermore, the sign of the optical activity (relative to the $12.1 \mathrm{wt} \%$ data) is opposite for concentrations above and below 12.1 wt. \%.

The theory of short range order in the isotropic phase of chiral nematic liquid crytals predicts that the optical activity $\phi$ should vary as follows:

$$
\phi=\frac{\pi k_{B} q_{0}}{12 \epsilon_{0} \lambda^{2} \sqrt{a_{0} b}}\left[1+\frac{c}{2 b}\right]^{-3 / 2} \frac{T}{\sqrt{T-T_{1}^{*}}},
$$

where $k_{B}$ is Boltzmann's constant, $\epsilon_{0}$ is the permeability of free space, $\lambda$ is the wavelength of the light in the sample, $a_{0}$ and $b$ are the same phenomenological parameters as in Eq.

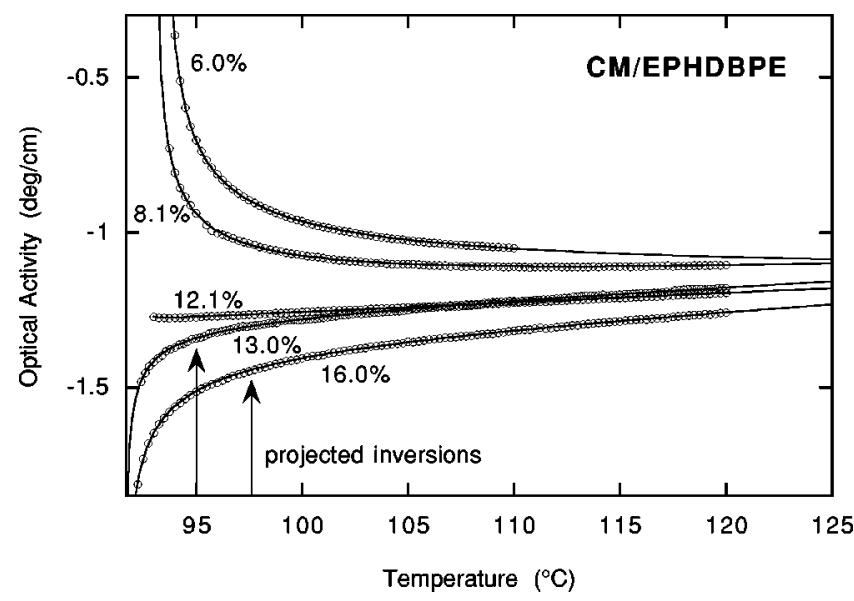

FIG. 5. Optical activity in the isotropic phase for five CMEPHDBPE mixtures. The lines are least square fits to Eq. (13). The arrows point to where extrapolation of the helix inversion line in the chiral nematic phase intersects the data.

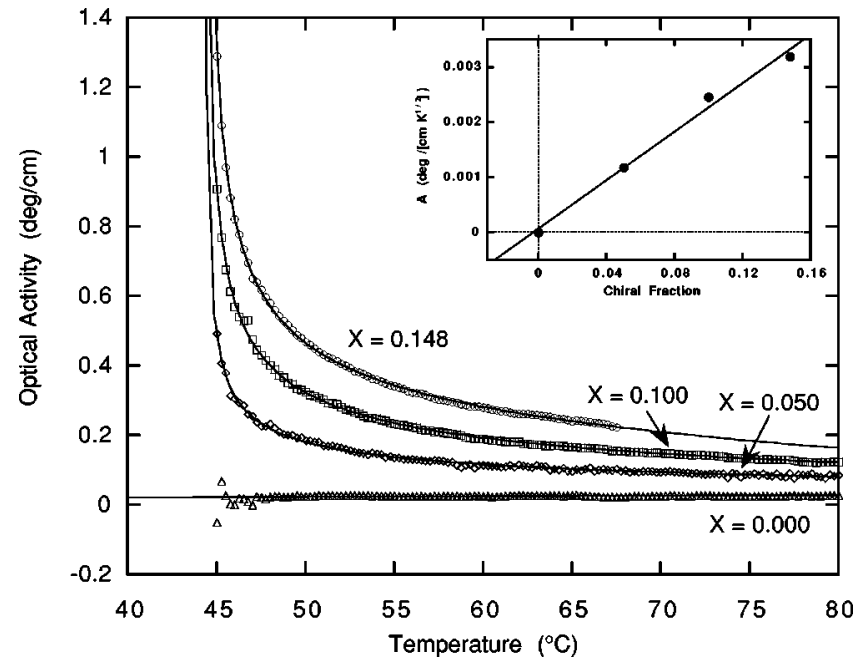

FIG. 6. Optical activity in the isotropic phase for four chiralracemic CE6 mixtures. The lines are least square fits to Eq. (13). The inset shows the dependence of the parameter A in Eq. (13) obtained from these fits on the fraction of S-CE6 in the S-CE6racemic-CE6 mixtures. The line in the inset is a linear least square fit to the data.

(2), $c$ is an additional phenomenological parameter, and $T_{1}^{*}$ is the second order transition temperature for the structural mode most responsible for optical activity in the isotropic phase [20]. If the assumption is made that the values of the phenomenological constants do not change significantly due to the small differences in CM concentration, then the factor multiplying the temperature dependence is proportional to the chirality $q_{0}$. For this reason, the optical activity data were fitted to

$$
\phi=\frac{A T}{\sqrt{T-T_{1}^{*}}}+B+C T,
$$

where the last two terms allow for a slightly temperature dependent "molecular" contribution to the optical activity ( $T$ is in kelvin). These fits are extremely good and are shown in Fig. 5.

Theoretically, the fitting parameter A should be proportional to the chirality in the isotropic phase $q_{0}$. To check this experimentally, the optical activity of chiral-racemic mixtures of CE6 with chiral fractions $0.000,0.050,0.100$, and 0.148 were performed. The results are shown in Fig. 6, along with fits to Eq. (13). Since previous measurements on chiralracemic mixtures of CE6 have revealed that the chirality in the chiral nematic phase at the isotropic phase transition is proportional to the chiral fraction [26], the inset shows a plot of the values of A determined from these fits versus chiral fraction, verifying that the parameter $\mathrm{A}$ is proportional to $q_{0}$ in this system.

Figure 7 shows a plot of A versus $q_{0}$ in the chiral nematic phase at the phase transition temperature to the isotropic phase for the five $\mathrm{CM} /(S, S)$-EPHDBPE mixtures. The linear relationship between these two quantities coupled with the fact that the fits in Fig. 5, where the chirality for each mixture was assumed to be temperature independent, suggests that the chirality in the isotropic phase for a liquid crystal is constant and proportional to the chirality in the chiral nem- 


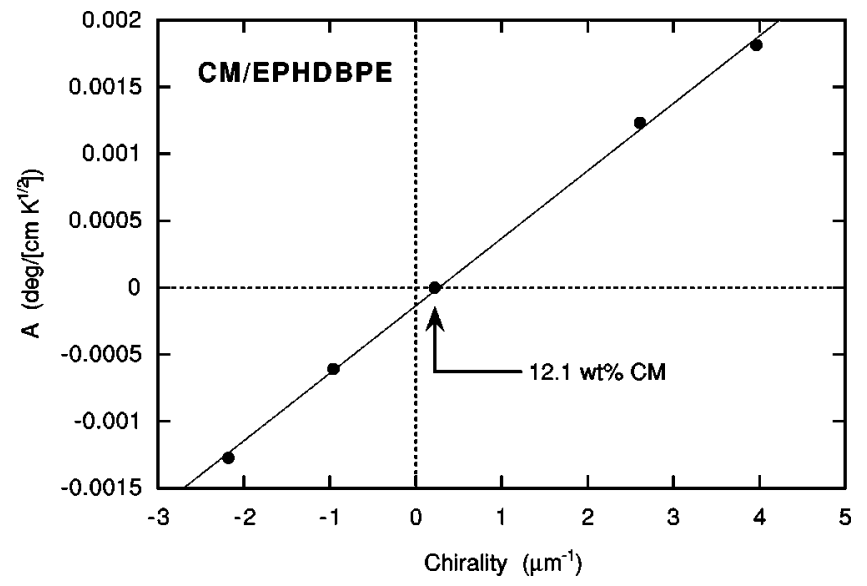

FIG. 7. The dependence of the parameter A in Eq. (13), obtained from the fits shown in Fig. 5, on the chirality in the chiral nematic phase at the transition to the isotropic phase.

atic phase at the phase transition temperature. For this reason, the region above the transition line in Fig. 2 is labeled with one handedness for CM concentrations less than 12 wt. \% and with the other handedness for CM concentrations greater than 12 wt. $\%$.

\section{DISCUSSION}

The abrupt change in the slope of the helix inversion line at the chiral nematic to isotropic phase transition is an important finding. Clearly the origin of chirality cannot be mainly in the short range molecular correlations, since these should not change to a great extent in crossing the transition. Such molecular correlations could very well be important, but obviously the amount of long range orientational order plays an important role.

In addition, although the molecular field and phenomenological theories can explain helix inversion in the chiral nematic phase, they predict an abrupt change in the chirality at the transition that is not supported by experiment. Specifically, they predict that the chirality in the isotropic phase of all mixtures should be of one sign and not proportional to the chirality in the chiral nematic phase (see Tables I and II). This is not at all surprising, as neither of these theories takes into account the short range molecular correlations that occur near the transition in both phases but are the only order present in the isotropic phase.
The optical activity measurements are only capable of determining a quantity that is proportional to the chirality in the isotropic phase. A direct measurement of the chirality in the isotropic phase is needed, but such measurements are difficult. Light scattering experiments are capable of measuring the chirality in the isotropic phase, and such an experiment has been performed in one liquid crystal system [27]. For this system, the transition to the isotropic phase was from the third blue phase $\left(\mathrm{BP}_{\mathrm{III}}\right)$ not the chiral nematic phase. The $\mathrm{BP}_{\mathrm{III}}$ to isotropic transition ends at a critical point [23], and for the mixture studied by light scattering the critical point was probably not far away. So the finding that the chirality was continuous (to within experimental error) across the $\mathrm{BP}_{\mathrm{III}}$-ISO transition is not surprising. Unfortunately, this result cannot be assumed to hold for the CM/ $(S, S)$-EPHDBPE mixtures, where the transition is from the chiral nematic phase and is much more discontinuous. Light scattering experiments to measure the chirality of the isotropic phase in a system without blue phases are underway at this time. The results gathered so far seem to indicate that if a discontinuity in the chirality exists at the chiral nematic to isotropic transition, it is not large and certainly much smaller than anything predicted by the two theories. In fact, at this point a continuous chirality across the transition cannot be ruled out.

\section{CONCLUSIONS}

Investigation of a system with a helix inversion line is capable of revealing in a detailed way some of the complex chiral behavior of liquid crystals. The change in temperature dependence of the chirality and the proportionality of the chirality across the chiral-nematic-isotropic-phase transition are findings that call for a theoretical explanation. Although there is no reason to suspect that the $\mathrm{CM} /(S, S)$-EPHDBPE mixture is a special case, it is important to ascertain just how general this behavior is. Only additional experiments on different systems can answer this question.

\section{ACKNOWLEDGMENTS}

This research was supported by the National Science Foundation under Grant No. DMR-9224617 to Swarthmore College and Laboratory for Reseach on the Structure of Matter of the University of Pennsylvania. Useful discussions with D. Kruerke and T. Lubensky are gratefully acknowledged.
[1] B. W. van der Meer, G. Vertogen, A. J. Dekker, and J. G. J. Ypma, J. Chem. Phys. 65, 3935 (1976).

[2] J. P. Straley, Phys. Rev. A 14, 1835 (1976).

[3] G. T. Evans, Mol. Phys. 77, 969 (1992).

[4] M. A. Osipov, B. T. Pickup, and D. A. Dunmur, Mol. Phys. 84, 1193 (1995).

[5] R. A. Pelcovits, Liq. Cryst. 21, 361 (1996).

[6] A. Ferrarini, G. J. Moro, and P. L. Nordio, Phys. Rev. E 53, 681 (1996); Mol. Phys. 87, 485 (1996)

[7] A. B. Harris, R. D. Kamien, and T. C. Lubensky, Phys. Rev. Lett. 78, 1476 (1997); 78, 2867E (1997).

[8] R. Memmer and F. Janssen, Liq. Cryst. 24, 805 (1998).
[9] H. Stegemeyer, K. Siemensmeyer, W. Scurow, and L. Appel, Z. Naturforsch. A 44, 1127 (1989).

[10] A. J. Slaney, I. Nishiyama, P. Styring, and J. W. Goodby, J. Mater. Chem. 2, 805 (1992).

[11] H.-G. Kuball, Th. Muller, and H.-G. Weyland, Mol. Cryst. Liq. Cryst. 215, 271 (1992).

[12] I. Dierking, F. Giesselmann, P. Zugemaier, W. Kuczynski, S. T. Lagerwall, and B. Stebler, Liq. Cryst. 13, 45 (1993).

[13] P. Styring, J. D. Vuijk, I. Nishiyama, A. J. Slaney, and J. W. Goodby, J. Mater. Chem. 3, 399 (1993).

[14] I. Dierking, F. Giesselmann, P. Zugenmaier, K. Mohr, H. Zaschke, and W. Kuczynski, Z. Naturforsch. A 49, 1081 
(1994).

[15] I. Dierking, F. Giesselmann, P. Zugenmaier, K. Mohr, H. Zaschke, and W. Kuczynski, Liq. Cryst. 18, 443 (1995).

[16] H.-G. Kuball, Th. Muller. H. Bruning, and A. Schoenhofer, Mol. Cryst. Liq. Cryst. 261, 205 (1995).

[17] I. Dierking, F. Giesselmann, and P. Zugenmaier, Mol. Cryst. Liq. Cryst. 281, 79 (1996).

[18] W. Maier and A. Saupe, Z. Naturforsch. A 13, 564 (1958); 14, 882 (1959); 15, 287 (1960).

[19] P. G. DeGennes, Phys. Lett. A 30, 454 (1969); Mol. Cryst. Liq. Cryst. 12, 193 (1971).

[20] D. Bensimon, E. Domany, and S. Shtrikman, Phys. Rev. A 28, 427 (1983).
[21] P. J. Collings and M. Hird, Introduction to Liquid Crystals: Chemistry and Physics (Taylor \& Francis, London, 1997), p. 34.

[22] K. H. Yang, J. Appl. Phys. 64, 4780 (1988).

[23] Z. Kutnjak, C. W. Garland, C. G. Schatz, P. J. Collings, C. J. Booth, and J. W. Goodby, Phys. Rev. E 53, 4955 (1996).

[24] N. V. Madhusudana and R. Pratibha, Mol. Cryst. Liq. Cryst. 89, 249 (1982).

[25] B. Chu, C. S. Bak, and F. L. Lin, Phys. Rev. Lett. 28, 111 (1972).

[26] D. K. Yang and P. P. Crooker, Phys. Rev. A 35, 4419 (1987).

[27] E. P. Koistinen and P. H. Keyes, Phys. Rev. Lett. 74, 4460 (1995). 